\title{
Quantitative trait loci for flowering time and morphological traits in multiple populations of Brassica rapa
}

\author{
Ping Lou ${ }^{1, *}$, Jianjun Zhao ${ }^{1,2,3,5,6, *}$, Jung Sun Kim ${ }^{4}$, Shuxing Shen ${ }^{2}$, Dunia Pino Del Carpio ${ }^{1}$, Xiaofei Song ${ }^{2}$, \\ Mina Jin ${ }^{4}$, Dick Vreugdenhil ${ }^{6}$, Xiaowu Wang ${ }^{3}$, Maarten Koornneef ${ }^{5,7}$ and Guusje Bonnema ${ }^{1, \dagger}$ \\ ${ }^{1}$ Laboratory of Plant Breeding, Wageningen University, Droevendaalsesteeg 1, 6708 PB Wageningen, \\ The Netherlands \\ ${ }^{2}$ Horticultural College, Hebei Agricultural University, Hebei Province, Baoding 071001, China \\ ${ }^{3}$ Institute of Vegetables and Flowers, Chinese Academy of Agricultural Sciences, 12 Zhongguancun Nandajie, \\ Beijing 100081, China \\ ${ }^{4}$ Brassica Genomics Team, National Institute of Agricultural Biotechnology, 225 Seodun-Dong, Suwon, 441-707, \\ South Korea \\ ${ }^{5}$ Laboratory of Genetics, Wageningen University, Arboretumlaan 4, 6703 BD Wageningen, The Netherlands \\ ${ }^{6}$ Laboratory of Plant Physiology, Wageningen University, Arboretumlaan 4, 6703 BD Wageningen, The Netherlands \\ ${ }^{7}$ Max Planck Institute for Plant Breeding Research, Carl von Linné Weg 10, 50829 Cologne, Germany
}

Received 29 May 2007; Revised 18 September 2007; Accepted 20 September 2007

\begin{abstract}
Wide variation for morphological traits exists in Brassica rapa and the genetic basis of this morphological variation is largely unknown. Here is a report on quantitative trait loci (QTL) analysis of flowering time, seed and pod traits, growth-related traits, leaf morphology, and turnip formation in $B$. rapa using multiple populations. The populations resulted from crosses between the following accessions: Rapid cycling, Chinese cabbage, Yellow sarson, Pak choi, and a Japanese vegetable turnip variety. A total of 27 QTL affecting 20 morphological traits were detected, including eight QTL for flowering time, six for seed traits, three for growth-related traits and 10 for leaf traits. One major QTL was found for turnip formation. Principal component analysis and co-localization of QTL indicated that some loci controlling leaf and seed-related traits and those for flowering time and turnip formation might be the same. The major flowering time QTL detected in all populations on linkage group R02 colocalized with BrFLC2. One major QTL, controlling turnip formation, was also mapped at this locus. The genes that may underly this QTL and comparative
\end{abstract}

analyses between the four populations and with Arabidopsis thaliana are discussed.

Key words: Brassica rapa, comparative mapping, flowering time, morphological traits, multiple populations, quantitative trait loci.

\section{Introduction}

Brassica rapa is an important species of the genus Brassica, which provides both rapeseed oil, fodder, and vegetables contributing to the world economy and to the health of people as a source of beneficial nutrients. During the long history of breeding and selection, a variety of forms have been selected for use as oilseeds, leafy vegetables and turnips.

Up to now, limited information is available on the inheritance of morphological traits in this species. Genetic studies aiming at the identification of loci controlling morphological variation have illustrated the complex genetic control of the many quantitatively inherited traits in B. rapa (Song et al., 1995; Yu et al., 2003). The study of the genetics of the morphology of the curd in Brassica

\footnotetext{
* These authors contributed equally to this work.

† To whom correspondence should be addressed. E-mail: guusje.bonnema@wur.nl
} 
oleracea (Lan and Paterson, 2000; Sebastian et al., 2002), is an example of the analysis of the genetic basis for morphological and developmental traits in other Brassica species. A recent review on Arabidopsis (Koornneef et al., 2004) indicated that co-location of QTL for floral, leaf morphology, and other growth-related traits provided clear evidence for a modular genetic architecture, where similar loci control a number of related processes. Modularity implies that the quantitative expression of particular traits tends to vary in a co-ordinated and structured manner. One of the mechanisms that can cause modularity is genetic correlation among traits, due to pleiotropy or extremely tight linkage (Conner, 2002). Linking the studies of Arabidopsis with Brassica is feasible nowadays because the syntenic relationships are better established (Kim et al., 2006; Parkin et al., 2005; Suwabe et al., 2006).

Among the agronomic traits, flowering time is one of the most important traits and wide variation exists among $B$. rapa. It is affected by the growing season and thus varieties are bred for specific geographical regions and seasons. In the Brassicaceae family, many studies have examined QTL affecting flowering time in different environments using different populations. In Arabidopsis, the largest difference in flowering time among ecotypes appears to be due to allelic variation at the FLC (Flowering locus $C$ ) and FRI (FRIGIDA) loci (Koornneef et al., 2004; Engelmann and Purugganan, 2006). In B. oleracea, two or three genome regions containing QTL for flowering time have been identified (Kennard et al., 1994; Bohuon et al., 1998; Rae et al., 1999). Recently four FLC copies were isolated in B. oleracea: BoFLC2 probably contributes to the control of flowering time, while $B o F L C 1, B o F L C 3$, and BoFLC5 were found to be unlinked to the QTLs controlling flowering time (Okazaki et al., 2007). In B. nigra, Lagercrantz's group observed that a genomic region, which is co-linear with the top of chromosome 5 of Arabidopsis, was associated with flowering time variation and suggested $C O$ as a likely candidate gene for this flowering time QTL. Furthermore, they compared the genetics of flowering time in four Brassica species and concluded that for $C O$, and not for $F L C$, duplicated copies were likely candidates for flowering time QTL (Lagercrantz et al., 2002; Osterberg et al., 2002).

In B. rapa, several QTL (VFR1, VFR2, and VFR3; FR1, $F R 2$, and $F R 3$ ) for flowering time were identified in an $\mathrm{F}_{2}$ and a recombinant inbred line population derived from a cross between an annual and a biennial oil type (Teutonico and Osborn, 1994; Osborn et al., 1997). VFR2 was estimated to have a large effect and was suggested to be homologous to FLC of Arabidopsis. A further study confirmed that $V F R 2$ locates at the BrFLCl locus, FRI at the position of $B r F L C 2$ and $F R 2$ at BrFLC5; VFRI was mapped on $\mathrm{R} 02$ close to a region syntenic to the $M A F$
(MADS Affecting Flowering) region at the bottom of chromosome 5 in Arabidopsis. These three B. rapa flowering time genes $B r F L C 2, B r F L C 3$, and $B r F L C 1$ were assigned to linkage groups R02, R03, and R10, respectively (Kole et al., 2001; Schranz et al., 2002; Kim et al., 2006).

Bolting time has also been analysed under different conditions in a population derived from a cross between two heading Chinese cabbages, and 10 QTL located on six linkage groups were identified (Ajisaka et al., 2001; Nishioka et al., 2005; Zhang et al., 2006). However, these linkage groups were not assigned to the reference linkage groups and therefore it is not possible to compare these QTL to other flowering time QTL. In general, it seems that the multiple copies of Brassica genes homologous to flowering time genes, especially those at the top of chromosome 5 of Arabidopsis such as FLC and $\mathrm{CO}$, contribute to the wide variation in flowering time in the genus Brassica.

All the research described above used oil-type $B$. rapa for mapping flowering time genes and it is interesting to know what is the genetic variation for flowering time in the other $B$. rapa types. In a previous study, the relationship between accessions was revealed by AFLP fingerprinting in a large collection of B. rapa (Zhao et al., 2005). One finding was that genetic distance was more related to geographical origin (East Asia versus Europe) than to the different morpho-types. This prompted further investigation of the genetic relationships by crossing genotypes with different morpho-types and geographical origins.

In this study, a number of segregating populations with parents selected from the three main groups that are distinguished in B. rapa (the oil-, leafy-, and turnip types) were used to dissect plant morphology genetically. The aim was to detect QTL for morphological traits using multiple populations derived from three different main $B$. rapa morpho-types.

\section{Materials and methods}

\section{Plant materials and growing conditions}

Three different types of populations were developed from wide crosses between $B$. rapa accessions. The parental accessions were selected based on their origins, morphological types, and their AFLP patterns, which were described in a previous study (Zhao et al., 2005).

The $\mathrm{F}_{2 / 3}$ (RC-CC) population (178 $\mathrm{F}_{2}$ plants) was produced from a cross between a Rapid cycling line RC-144 (accession number: FIL501) and a vegetable type Chinese cabbage line CC-156 (cultivar: Huang Yang Bai; accession number: VO2A0030). Both $\mathrm{F}_{2}$ and $\mathrm{F}_{3}$ were used to evaluate flowering time, plant height, leaf traits, and seed weight in three experiments (Table 1). In the case of the $\mathrm{F}_{3}$ lines 10 plants per line were grown for phenotypic analysis. Double haploid (DH) populations were developed from crosses between the oil type Yellow sarson YS-143 (accession number: 
FIL500) and the vegetable types Pak choi PC-175 (cultivar: Nai Bai Cai; accession number: VO2B0226) and Vegetable turnip VT-115 (cultivar: Kairyou Hakata; accession number: CGN15199). A total of 135 lines including 71 lines from population DH-38 (PC$175 \times$ YS-143), 64 lines from population DH-30 (VT-115×YS-143) were analysed for flowering time, leaf traits, seed colour, and seed pod traits. DH-30 was also used to evaluate turnip formation. Five plants per $\mathrm{DH}$ line were grown in pots for the greenhouse experiment, and three plants per DH lines with two replications for the open field experiment.

An additional backcross (BC1) population of 136 plants [(VT$115 \times$ YS-143) $\times$ VT-115] was developed from a cross between one $\mathrm{F}_{1}$ plant (VT-115 $\times$ YS-143) and one plant of parental accession VT115 . Both flowering time and turnip formation were analysed in this population.

\section{Trait analysis}

In total, 22 traits related to flowering, seed, growth (plant height and branch number), leaf and turnip formation were recorded in 1-4 populations. The traits and their description are shown in Table 2.
The leaf characteristics were scored on a fully developed leaf before flowering stage at a fixed date and subdivided in lamina length (LL), lamina width (LW), petiole length (PL), and leaf edge shape (LES) as illustrated in Fig. 1a. The values of leaf area (LA), LL, LW, and PL in DH-38 and DH-30 were obtained by analysing the leaf photographs using Scion Image (Scion Corparation, MD, USA, http://rsb.info.nih.gov/nih-image), where the leaf photographs were digitally processed with the Irfanview program (http:// www.irfanview.com). The values of $\mathrm{LL}$ and $\mathrm{LW}$ of $\mathrm{F}_{3}$ plants were measured using a ruler. The mature and dried seedpod traits were measured once on harvested siliques in the greenhouse experiment of spring 2005. Seedpod characteristics are shown in Fig. 1a.

\section{Map alignment and target markers for flowering time genes}

Linkage analysis and map construction for $\mathrm{F}_{2 / 3}$, DH-38, DH-30, and $\mathrm{BC} 1$ were carried out using the program Joinmap 3.0 (Van Ooijen and Voorrips, 2001) (G Bonnema, unpublished data). All the maps were aligned based on common SSR markers and compared with the JWF3p map available on the Brassica rapa Genome Project (BrGP) website (www.Brassica-rapa.org). Seven flowering-timerelated genes or their flanking SSR markers were selected from the

Table 1. Experimental setup for multiple populations

\begin{tabular}{|c|c|c|c|c|c|c|}
\hline \multirow[t]{2}{*}{ Trial } & \multirow[t]{2}{*}{ Sowing date } & \multicolumn{3}{|l|}{ Conditions } & \multirow[t]{2}{*}{ Location } & \multirow[t]{2}{*}{ Population } \\
\hline & & Types & Day length & $\begin{array}{l}\text { Temperature } \\
(\max / \mathrm{min})\end{array}$ & & \\
\hline $04 \mathrm{sp}$ & Jan. 2004 & Non-heated greenhouse & Natural day length (12-14 h) & $5^{\circ} \mathrm{C} / 25^{\circ} \mathrm{C}$ & Beijing, China & $\mathrm{F}_{2}$ (178 plants) \\
\hline $05 \mathrm{spcn}$ & Jan. 2005 & Non-heated greenhouse & Natural day length $(12-14 \mathrm{~h})$ & $10^{\circ} \mathrm{C} / 30^{\circ} \mathrm{C}$ & Beijing, China & $\mathrm{F}_{3}$ (125 lines) \\
\hline 05 wi & Sep, 2005 & Heated greenhouse & Controlled day length $(16 \mathrm{~h})$ & $18^{\circ} \mathrm{C} / 24^{\circ} \mathrm{C}^{a}$ & Wageningen, Netherlands & $F_{3}(115$ lines $)$ \\
\hline 04wi & $24 / 09 / 2004$ & Heated greenhouse & Controlled day length $(16 \mathrm{~h})$ & $18^{\circ} \mathrm{C} / 24^{\circ} \mathrm{C}^{a}$ & Wageningen, Netherlands & DH (135 lines) \\
\hline $05 \mathrm{spnl}$ & $03 / 03 / 2005$ & Heated greenhouse & Controlled day length (16h) & $18^{\circ} \mathrm{C} / 24^{\circ} \mathrm{C}^{a}$ & Wageningen, Netherlands & DH (135 lines) \\
\hline 05au & $15 / 07 / 2005$ & Open field & Natural day length (14-16 h) & $7^{\circ} \mathrm{C} / 30{ }^{\circ} \mathrm{C}$ & Wageningen, Netherlands & $\begin{array}{l}\text { DH (135 lines) } \\
\text { BC (136 plants) }\end{array}$ \\
\hline
\end{tabular}

${ }^{a}$ Here night/day temperatures settings are listed. Since there was no cooling in the greenhouse, temperatures during daytime were occasionally higher than $24{ }^{\circ} \mathrm{C}$.

Table 2. List of traits analysed

\begin{tabular}{|c|c|c|c|c|}
\hline Trait type & Trait name & ABS & Trait description & Scale \\
\hline Flowering time & Flowering time & FL & Days from sowing to appearance of the first open flower & days \\
\hline \multirow{5}{*}{ Seed-related trait } & Seed pod length & SPL & Length between pedicel of silique and top of beak (Fig. 1) & $\mathrm{mm}$ \\
\hline & Seed pod width & SPW & Width at the lengthwise midpoint of each silique (Fig. 1) & $\mathrm{mm}$ \\
\hline & Beak length & SBL & Length between the top of silique and the top of beak (Fig. 1) & $\mathrm{mm}$ \\
\hline & Seed colour & SC & Scored as 1 , yellow; 2 , yellow brown; 3 , light brown; 4 , brown; 5 , dark brown & $1-5$ \\
\hline & Seed weight & SW & $\begin{array}{l}\text { The mean seed weight, obtained by weighting } 2 \text { to } 5 \text { seed lots each of } \\
20 \text { seeds }\end{array}$ & $\mathrm{mg}$ \\
\hline \multirow[t]{2}{*}{ Growth-related trait } & Plant height & PH & Height from ground to the apical point of plant at flowering stage & $\mathrm{cm}$ \\
\hline & Branches & $\mathrm{PB}$ & The number of main branches & number \\
\hline \multirow[t]{9}{*}{ Leaf trait } & Leaf edge shape & LES & Scored as 1 , entire; 2 , slightly serrated; 3 , intermediate serrated; 4 , much serrated & $1-4$ \\
\hline & Leaf trichomes & LT & Hair on leaf surface scored as 0 -hair absent, 1 -hair present before flowering & $0-1$ \\
\hline & Leaf number & $\mathrm{LN}$ & Number of leaves before flowering & number \\
\hline & Leaf lobes & LB & Scored as 0 , absent; 1 , present & $0-1$ \\
\hline & Lamina length & LL & From base of petiole to tip of lamina (Fig. 1) & $\mathrm{cm}$ \\
\hline & Lamina width & LW & Lamina width at the widest point (Fig. 1) & $\mathrm{cm}$ \\
\hline & Petiole length & PL & From base of petiole to bottom of lamina (Fig. 1) & $\mathrm{cm}$ \\
\hline & Leaf area & LA & The whole surface of full leaf & $\mathrm{cm}^{2}$ \\
\hline & Leaf index & LI & Ratio of LL to LW, LL/LW & ratio \\
\hline \multirow[t]{5}{*}{ Turnip trait } & Turnip formation & $\mathrm{TF}$ & Qualitative score of turnip formation (1-4 scale, Fig. 1) & $1-4$ \\
\hline & Turnip shoots & TS & Number of shoots on the turnip (Fig. 1) & number \\
\hline & Turnip length & TL & Length from the top to bottom of turnip, measured by vernier caliper (Fig. 1) & $\mathrm{mm}$ \\
\hline & Turnip width & TWi & Width at the widest point, measured by vernier caliper (Fig. 1) & $\mathrm{mm}$ \\
\hline & Turnip weight & TWe & The mean weight of each turnip after harvesting & $\mathrm{g}$ \\
\hline
\end{tabular}


1 a

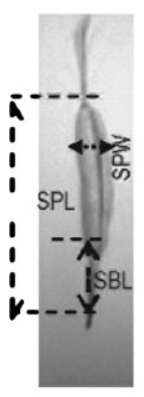

A

$1 \mathrm{~b}$

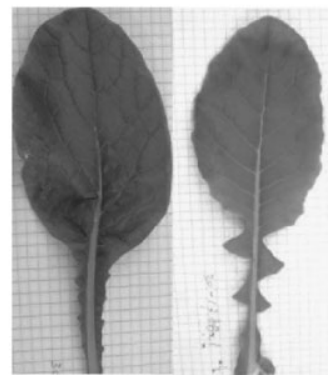

B
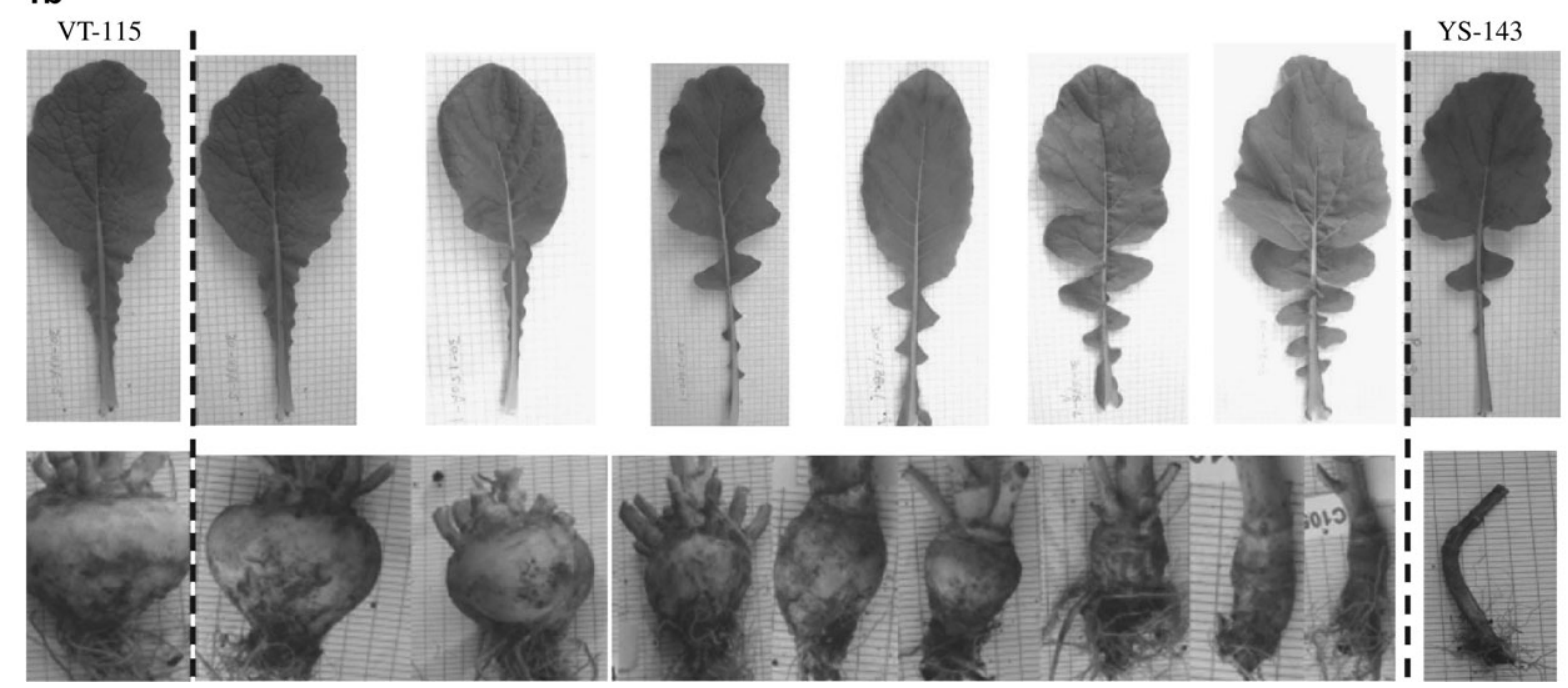

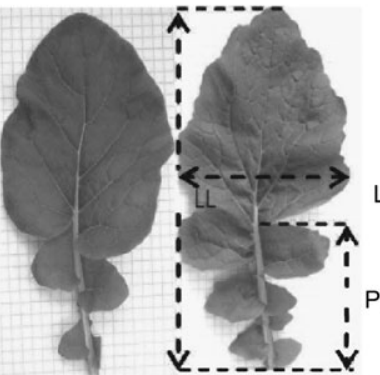

B

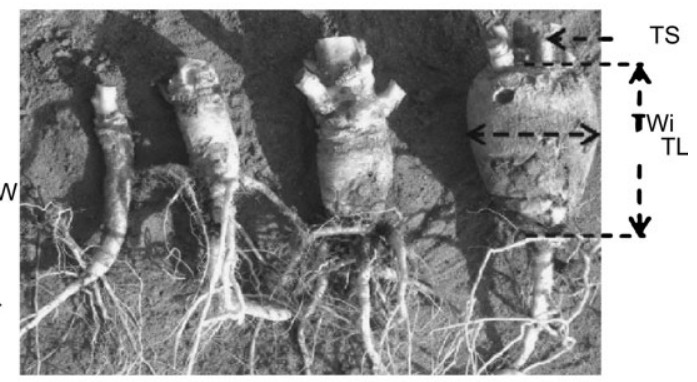

C

Fig. 1. Pictorial representation of measurement and variation of parental lines and populations. (a) Measurement of seedpod (A), leaf (B), and turnip traits (C). Leaf edge shape (LES) classifications are indicated in B from left to right 1-4. Turnip formation classifications are indicated in C from left to right 1-4. For detail descriptions see Table 2. (b) An example of the variation for leaf traits and turnip traits of parental accessions VT-115 and YS143 and selected individuals from population DH-30 (for leaf traits) and BC1 (for turnip trait) displaying all phenotypic variations.

JWF3p map based on the QTL positions of flowering time detected in this study (Table 3).

\section{Statistical analysis of phenotypic data}

Statistical analysis for distribution and correlation were performed in Genstat 8.1. We also conducted a Principal Component Analysis (PCA) in Genstat 8.1 on the line means for the flower, seed, leaf, and turnip-related traits to evaluate the correlations between the various traits.

\section{QTL analysis}

QTL analysis was done for each experiment and population separately. The computer software MAPQTL 5.0 was used to perform QTL analysis using both interval mapping (IM) and multiple-QTL model mapping (MQM) methods (Van Ooijen, 2004). The analysis started with the interval-mapping test to find putative QTL. MQM analysis was then performed to locate QTL precisely after the automatic selection of cofactors in the vicinity of QTL. Only significant markers at $P<0.02$ were used as cofactors in the multiple QTL detection. A map interval of $5 \mathrm{cM}$ was used for both IM and MQM analyses. A permutation test was applied to each data set (1000 repetitions) to decide the LOD (Logarithm of Odds) thresholds $(P=0.05)$. LOD values of 2.9 for $\mathrm{F}_{2 / 3}, 2.0$ for $\mathrm{DH}$ -
38, DH-30, and $\mathrm{BC} 1$ were used as a significance threshold for the presence of a candidate QTL. For each QTL, two-LOD support intervals were established as approximately $95 \%$ confidence intervals. Graphic representations of maps were produced by Mapchart software (Voorrips, 2002).

\section{Results}

\section{Natural variation in flowering time and morphological traits}

The five parental lines belong to different morpho-types and displayed variation for flowering time, seed traits, plant height, leaf traits, and turnip traits (Table 4; Fig. 1). Transgression beyond the parental values within the analysed populations was observed for most of the measured traits including those for which parental values hardly differed, such as seed pod width (SPW) and leaf width (LW) in DH-38. The flowering time ranged from 17 $\mathrm{d}$ to $132 \mathrm{~d}$ within populations, depended on growing season, the parental genotypes, and locations, and was transgressive in both directions in DH-38, DH-30, and 
QTL of flowering time and morphological traits in B. rapa 5 of 12

Table 3. Markers from JWF3p reference map for comparative study

\begin{tabular}{llll}
\hline Flowering time related genes & Copies & R-group & Gene target and flanking markers in JWF3p map \\
\hline FLOWERING LOCUS C & FLC1 & R10 & BrFLC1;KS50150;KS50190;KS50240 \\
& FLC2 & R02 & BrFLC2;KS50320;KS50030;KS50170 \\
& FLC3 & R03 & KS20520;KS50270;KS50300;KS50140; KS50090;BRMS008;BRMS043 \\
FLOWERING LOCUS T & FLC5 & R03 & KS20470;BRMS043; \\
FLOWERING LOCUS CA & Fta & R07 & KS31001;KS31100;KS11440;KS11310;KS11280;BRMS040;BRMS036 \\
VERNALIZATION RESPONSE 2 & FCA & R02 & KS31020;KS50260;KS10420 \\
CONSTANS & RRN2 & R08 & KS40460;KS40282;KS50200;KS40660;KS40551 \\
MADS AFFECTING FLOWERING & CO & R03 & BRM2E02;BRMS088 \\
LEAFY & MAF & R02 & BrMAF2;BS30251;KS30180;KS30200;KS30040 \\
\hline & LFY & R06 & KS10280;KS10321;KS10420;KS50460;KS51090 \\
& & &
\end{tabular}

Table 4. Phenotypic values of parental lines and corresponding populations

\begin{tabular}{|c|c|c|c|c|c|c|c|c|c|c|c|c|c|}
\hline \multirow[t]{2}{*}{ Trait } & \multicolumn{4}{|l|}{$\mathrm{F}_{2 / 3}$} & \multicolumn{4}{|l|}{ DH-38 } & \multicolumn{3}{|l|}{ DH-30 } & \multicolumn{2}{|l|}{$\mathrm{BC} 1$} \\
\hline & CC-156 & RC-144 & Mean & Range & YS-143 & PC-175 & Mean & Range & VT-115 & Mean & Range & Mean & Range \\
\hline Flowering time $04 \mathrm{sp}$ & 107.8 & 40.8 & 70.9 & $40.0-122.0$ & - & - & - & - & - & - & - & - & - \\
\hline Flowering time 04 wi & $-^{a}$ & - & - & - & - & - & 86.3 & $39.0-128.0$ & - & 89.2 & $46.0-132.0$ & - & - \\
\hline Flowering time $05 \mathrm{sp}^{c}$ & 3.0 & 1.0 & 2.1 & $1.0-3.0$ & 45.0 & 54.0 & 58.2 & $35.0-87.0$ & 77.0 & 39.3 & $17.0-69.0$ & - & - \\
\hline Flowering time $05 \mathrm{wi}$ & $\mathrm{nd}^{b}$ & 27.4 & 46.6 & $27.3-66.2$ & - & - & - & - & - & - & - & - & - \\
\hline Flowering time $05 \mathrm{au}$ & - & - & - & - & 45.0 & 51.0 & 52.0 & $39.0-63.0$ & 59.0 & 50.9 & $39.0-69.0$ & 56.4 & $48.0-71.0$ \\
\hline Seed pot length & - & - & - & - & 70.0 & 45.3 & 43.0 & $20.4-67.4$ & 54.4 & 39.2 & $0.0-61.9$ & - & - \\
\hline Seed pot width & - & - & - & - & 5.0 & 5.7 & 4.9 & $2.6-7.9$ & 3.7 & 4.5 & $0.0-6.9$ & - & - \\
\hline Seed beak length & - & - & - & - & 20.5 & 6.4 & 9.4 & $2.5-18.8$ & 10.9 & 10.7 & 32.2 & - & - \\
\hline Seed colour & - & - & - & - & 1.0 & 5.0 & 2.9 & $1-5$ & 5.0 & 2.4 & $1-5$ & - & - \\
\hline Seed weight $04 \mathrm{sp}$ & 2.25 & 1.05 & 1.3 & $0.5-2.2$ & - & - & - & - & - & - & - & - & - \\
\hline Seed weight $05 \mathrm{sp}$ & - & 1.15 & 1.8 & $1.0-2.7$ & - & - & - & - & - & - & - & - & - \\
\hline Plant height & 16.5 & 19.1 & 33.3 & $10.0-67.5$ & - & - & - & - & - & - & - & - & - \\
\hline Plant branches & - & - & - & - & 6.0 & - & - & - & 8.0 & 7.5 & $3.8-15.0$ & 8.0 & $0.0-17.0$ \\
\hline Leaf edge shape $04 \mathrm{sp}$ & 2.0 & 3.0 & 2.3 & $1.0-4.0$ & - & - & - & - & - & - & - & - & - \\
\hline Leaf edge shape $05 w i$ & 2.0 & 3.0 & 1.9 & $1.0-3.9$ & - & - & - & - & - & - & - & - & - \\
\hline Leaf trichomes $04 \mathrm{sp}$ & 1.0 & 0.0 & 0.3 & $0.0-1.0$ & - & - & - & - & - & - & - & - & - \\
\hline Leaf trichomes $05 \mathrm{sp}$ & 1.0 & 0.0 & 0.3 & $0.0-1.0$ & - & - & - & - & - & - & - & - & - \\
\hline Leaf number $04 \mathrm{sp}$ & 9.0 & 5.0 & 6.8 & $4.0-13.0$ & - & - & - & - & - & - & - & - & - \\
\hline Leaf number $05 w i$ & 18.8 & 5.0 & 13.2 & $6.5-20.8$ & - & - & - & - & - & - & - & - & - \\
\hline Leaf lobes 05 wi & 0.0 & 1.0 & 0.4 & $0.0-1.0$ & - & - & - & - & - & - & - & - & - \\
\hline Leaf lobes $05 \mathrm{au}$ & - & - & - & - & 2.0 & 0 & 2.2 & $1.0-4.0$ & 0 & 1.9 & $1.0-4.0$ & - & - \\
\hline Leaf length & 33.4 & 4.8 & 24.7 & $8.8-44.8$ & 12.2 & 9.0 & 10.7 & $4.7-19.2$ & 32.9 & 13.9 & $6.4-25.7$ & - & - \\
\hline Lamina width & 21.5 & 1.7 & 10.7 & $4.3-19.8$ & 8.5 & 8.4 & 10.9 & $4.0-18.23$ & 9.4 & 7.6 & $4.3-11.3$ & - & - \\
\hline Petiole length & - & - & - & - & 12.3 & 3.8 & 5.7 & $0.1-13.0$ & 0.1 & 6.2 & $0.1-13.8$ & - & - \\
\hline Lamina area & - & - & - & - & 71.6 & 59.4 & 103.4 & $15.5-266.1$ & 160.6 & 68.8 & $27.7-160.9$ & - & - \\
\hline Leaf index & - & - & - & - & 41.0 & 29.7 & 39.5 & $15.6-67.5$ & 74.1 & 37.8 & $21.6-66.9$ & - & - \\
\hline Turnip formation & - & - & - & - & 1 & - & - & - & 4.0 & 2.0 & $1-3.8$ & - & - \\
\hline Turnip shoot & - & - & - & - & 0.0 & - & - & - & 5.0 & 2.6 & $0.0-14.0$ & 3.7 & $0.0-11.0$ \\
\hline Turnip length & - & - & - & - & 0.1 & - & - & - & 72.0 & 51.4 & $25.2-82.6$ & 57.7 & $35.0-80.0$ \\
\hline Turnip width & - & - & - & - & 2.0 & - & - & - & 22.0 & 14.0 & $5.18-39.0$ & 43.0 & $18.0-96.0$ \\
\hline Turnip weight & - & - & - & - & 5.4 & - & - & - & 243.0 & 11.3 & $0.77-53.6$ & 94.5 & $23.0-271.0$ \\
\hline
\end{tabular}

${ }^{a}$-, Not measured in the corresponding population.

${ }^{b}$ nd, No data because of no flowering at $130 \mathrm{~d}$ after sowing.

${ }^{c} 1$, early; 2 , middle; 3 , late.

$\mathrm{BC} 1$. In the RC-CC $\mathrm{F}_{2 / 3}$ population transgression for flowering time was only towards lateness as the RC-144 parent always had the shortest flowering time. The flowering time for the $\mathrm{F}_{2 / 3}$ and $\mathrm{DH}$ populations was, for each population, determined three times, with mean values and ranges differing considerably. However, a strong positive correlation between different experiments was observed within populations, with correlation coefficients $r=0.31-0.61$ in $\mathrm{F}_{2 / 3}, r=0.76-0.81$ in DH-38, and $r=0.87-$ 0.90 in DH-30.

Nine leaf traits were measured in the RC-CC $\mathrm{F}_{2 / 3}$ and the two DH populations before flowering. YS-143, the common parent of the two DH populations, had an average petiole length (PL) of $12.3 \mathrm{~cm}$; the $\mathrm{PC}-175$ parent had a short petiole of only $3.8 \mathrm{~cm}$, while VT- 115 had no petiole. Within populations the petiole length ranged from 
$0 \mathrm{~cm}$ to $13 \mathrm{~cm}$. Turnip-related traits, like weight, length, and width of the turnip, could only be measured in the $\mathrm{DH}-30$ and $\mathrm{BC} 1$ populations. In the $\mathrm{BC} 1$, all progenies had some degree of taproot thickening (Fig. 1b), and the mean value of turnip length $(57.5 \mathrm{~mm})$, turnip width (43.0 $\mathrm{mm}$ ), and turnip weight (94.5 g) was higher compared to plants of DH-30 grown in the open field in 2005.

All the morphological traits were grouped into five classes: flowering time measured in different growth seasons, seed-related traits, growth-related traits, leaf traits, and turnip traits (Table 2). A PCA analysis was performed for all 17 traits representing the five classes per population (see Supplementary Table S1 at $J X B$ online).

The positive correlation between flowering time and turnip traits was further analysed in the $\mathrm{BC} 1$ population (Fig. 2). Strong correlations were revealed between different turnip traits and flowering time in a backcross population (BC1) of 136 individuals. A number of significant genetic correlations were detected among the different turnip traits. The turnip width, length, and weight were positively correlated with each other (correlation coefficient $r=0.49-0.89$ ), but also with flowering time (correlation coefficient $r=0.57-0.67$ ).

\section{QTL mapping}

Flowering time: For flowering time, a total of eight QTL (FLQTLn) were identified on R01, R02, R03, R06, R07, $\mathrm{R} 08$, and $\mathrm{R} 10$ in four different populations evaluated in different growing seasons, three in $\mathrm{F}_{2 / 3}$, three in DH-38, four in $\mathrm{DH}-30$, and one in $\mathrm{BC} 1$ (Table 5; Fig. 3). In RC-CC $\mathrm{F}_{2 / 3}$, the explained variation per QTL was generally lower (8.9-24.6\%) than in the DH populations (13.4-59.3\%). A large percentage of phenotypic variance (17.7-59.3\%) was explained by FLQTL-2 on R02 in the DH populations. This QTL was detected in all populations, growing seasons, and conditions. FLQTL-6 was detected in both $\mathrm{F}_{2}$ and DH30 populations. However, other FLQTL were only detected in a single population and each QTL explained $15.1-19.7 \%$ of the phenotypic variation.

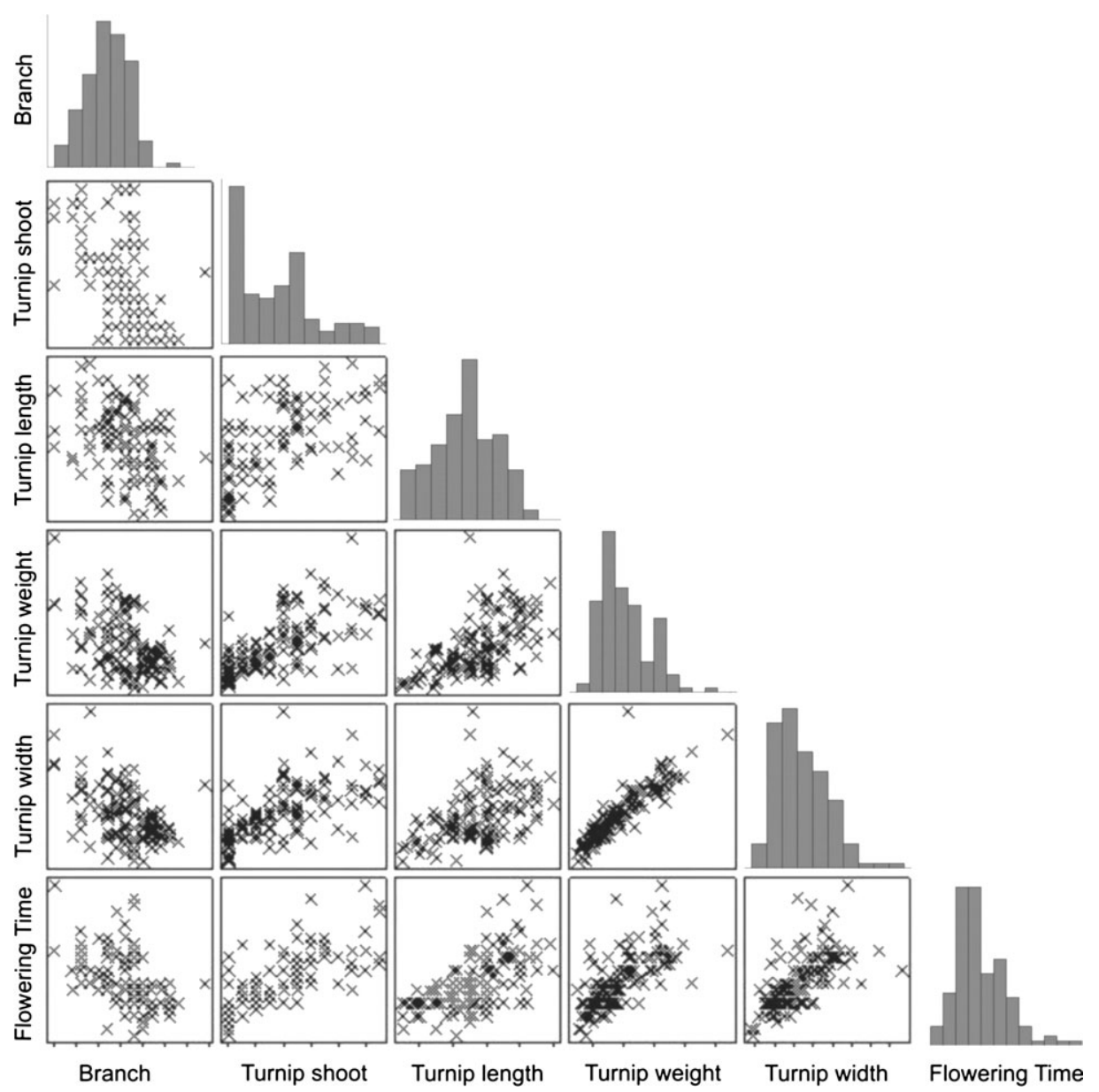

Fig. 2. Scatter plot matrix of turnip and flowering time traits generated from $B C 1$ population. The histograms along the diagonal provide a visual representation of the phenotypic variance for each of the traits. The off-diagonal scatter plots provide a visual representation of the correlation among the traits. 
Table 5. Results of QTL analyses of measured traits in four B. rapa populations

For trait abbreviation see Table 1; Exp: phenotypic variation explained. The position on the linkage group in Fig. 3 based on common SSR or AFLP markers. H-middle: higher-middle; L-middle: Lower middle. The accurate position for each population is indicated in Table S2.

\begin{tabular}{|c|c|c|c|c|c|c|}
\hline QTL & Trait & Population & Linkage group & Position $^{\mathrm{a}}$ & LOD & Exp\% \\
\hline FLQTL-1 & FL04wi & DH-30 & R01 & Middle & 2.25 & 13.4 \\
\hline FLQTL-2 & $\begin{array}{l}\text { FL04sp, FL04wi, } \\
\text { FL05sp, FL05wi, } \\
\text { FL05au }\end{array}$ & $\begin{array}{l}\text { F2/3, DH-38, DH-30, } \\
\text { BC1 }\end{array}$ & $\mathrm{R} 02$ & Top & $2.17-9.66$ & $8.9-59.3$ \\
\hline FLQTL-3 & FL05au & DH-38 & $\mathrm{R} 03$ & Bottom & 2.00 & 19.7 \\
\hline FLQTL-4 & FL05sp & $\mathrm{F} 2 / 3$ & R03 & Top & 5.01 & 9.3 \\
\hline FLQTL-5 & FL04wi & DH-30 & R06 & Middle & 2.42 & 18.3 \\
\hline FLQTL-6 & FL04sp, FL05au & $\mathrm{F} 2 / 3$ & R07 & H-middle & $4.26-6.02$ & $14.3-15.1$ \\
\hline FLQTL-7 & FL05au & DH-38 & $\mathrm{R} 10$ & Top & 2.70 & 17.9 \\
\hline FLQTL-8 & FL05sp & DH30 & R08 & Middle & 4.69 & 11 \\
\hline SPQTL-1 & SPL & DH-38 & R01 & Middle & 2.70 & 11.1 \\
\hline SPQTL-2 & SBL & DH-38 & R05 & Top & 3.87 & 20.3 \\
\hline SPQTL-3 & SPL, SBL, SPW & DH-38, DH-30 & R07 & H-middle & $4.68-6.39$ & $27.2-38.1$ \\
\hline SPQTL-4 & SBL & DH-30 & R09 & H-middle & 3.08 & 25.5 \\
\hline SCQTL-1 & SC & DH-38, DH-30 & R09 & Middle & $10.18-12.58$ & $61.7-65.5$ \\
\hline SWQTL-1 & SW04sp, SW05sp & $\mathrm{F} 2 / 3$ & $\mathrm{R} 03$ & Middle & $2.30-3.25$ & $10.2-11.6$ \\
\hline SWQTL-2 & SW05sp & $\mathrm{F} 2 / 3$ & R08 & H-middle & 4.22 & 17.6 \\
\hline PHQTL-1 & PH & $\mathrm{F} 2 / 3$ & R02 & H-middle & 6.17 & 15.7 \\
\hline PHQTL-2 & $\mathrm{PH}$ & $\mathrm{F} 2 / 3$ & R03 & Top & 3.00 & 8.9 \\
\hline PHQTL-3 & PH & $\mathrm{F} 2 / 3$ & R07 & H-middle & 9.43 & 23.9 \\
\hline LQTL-1 & $\begin{array}{l}\text { LB05wi, LN05wi, LL, } \\
\text { PL, LA }\end{array}$ & F2/3, DH-38 & R02 & H-middle & $2.20-6.12$ & $10.3-25.8$ \\
\hline LQTL-2 & LES04sp & $\mathrm{F} 2 / 3$ & R02 & L-middle & 3.32 & 6.5 \\
\hline LQTL-3 & $\begin{array}{l}\text { LES04sp, LES05wi, } \\
\text { LW, LI }\end{array}$ & F2/3, DH-38 & R03 & Bottom & $3.04-6.44$ & $20.5-26.4$ \\
\hline LQTL-4 & LW, LA, LI & F2/3, DH-30 & R05 & Middle & $2.33-3.16$ & $7.0-24.2$ \\
\hline LQTL-5 & LB05wi, LW, LL, LI & F2/3, DH-38, DH-30 & R06 & Middle & $2.12-6.07$ & $12.5-22.5$ \\
\hline LQTL-6 & LES04sp & $\mathrm{F} 2 / 3$ & R06 & L-middle & 3.47 & 9.1 \\
\hline LQTL-7 & $\begin{array}{l}\text { LL, LW, LB05wi, } \\
\text { LN04sp, LN05wi, PL, } \\
\text { LI }\end{array}$ & F2/3, DH-30 & R07 & H-middle & $3.41-6.03$ & $13.7-21.9$ \\
\hline LQTL-8 & LA & DH-38 & R08 & H-middle & 2.15 & 10.6 \\
\hline LQTL-9 & LW, LL & $\mathrm{F} 2 / 3$ & R08 & Bottom & $2.98-3.05$ & $9.4-11.2$ \\
\hline LQTL-10 & LW, LA, LL & DH-38 & R09 & H-middle & $2.00-2.53$ & $10.8-12.6$ \\
\hline TuQTL-1 & TF, TS, TL, TWi, TWe & DH-30, BC1 & R02 & Top & $4.74-7.08$ & $24.0-40.0$ \\
\hline
\end{tabular}

To investigate whether the same or different QTL positions were identified in the different populations, linkage maps were compared based on the common AFLP or SSR markers. In order to compare with the JWF3p map for flowering time QTL, 51 SSRs were screened against five parental lines and finally 10 loci were mapped to different linkage groups. The largest flowering time QTL, FLQTL-2 at the top of R02, was detected in the interval of ks50030 and P23M47115.6 in DH38 population and in this region $B r F L C 2$ was located according to the JWF3p map. In the $\mathrm{F}_{2 / 3}$ population FLQTL-2 also co-segregated with the SSR marker BrFLC2. There was strong overlap of the 2-LOD support intervals for this QTL across all the four populations. FLQTL-3 in DH-38 and FLQTL-4 in $\mathrm{F}_{2 / 3}$ are located on the same linkage group R03 but at different locations, FLQTL-3 located near marker BRMS043 and FLQTL-4 was mapped in the $F_{2 / 3}$ population at the top of R03, both of them far away from the flowering-time-related genes $B r F L C 5, B r F L C 3$, and CO. FLQTL-5 on R06 was near marker KS51082 where
$L F Y$ is located, and FLQTL-6 on R07 is close to one copy of the flowering time gene FT. FLQTL-8 on R08 located close to the VNR2 gene and FLQTL-7 colocalized with BrFLC1.

The FLQTLn detected in the populations were not always identical in different growing seasons and at different locations, indicating genotype $\times$ environment effects, which could reflect the effects of temperature and day-length response on the expression of these flowering time QTL. The major QTL FLQTL-2 on R02 was detected in all experiments (FL04sp, FL04wi, FL05sp, FL05wi, and FL05au) but other QTL were sensitive to environment; for example, FLQTL-5 on R06 was not detected in the open field experiment in DH-30 and FLQTL-7 on R10 was not detected in spring season 2005 in the greenhouse in DH-38.

In the $\mathrm{F}_{2 / 3}$ and $\mathrm{DH}$ populations, the earlier parent RC144 or YS-143 always contributed alleles that decreased flowering time except for FLQTL-4 and FLQTL-3 on R03, which were detected in spring 2005 in Beijing and autumn 2005 in Wageningen, respectively (Fig. 3). At 

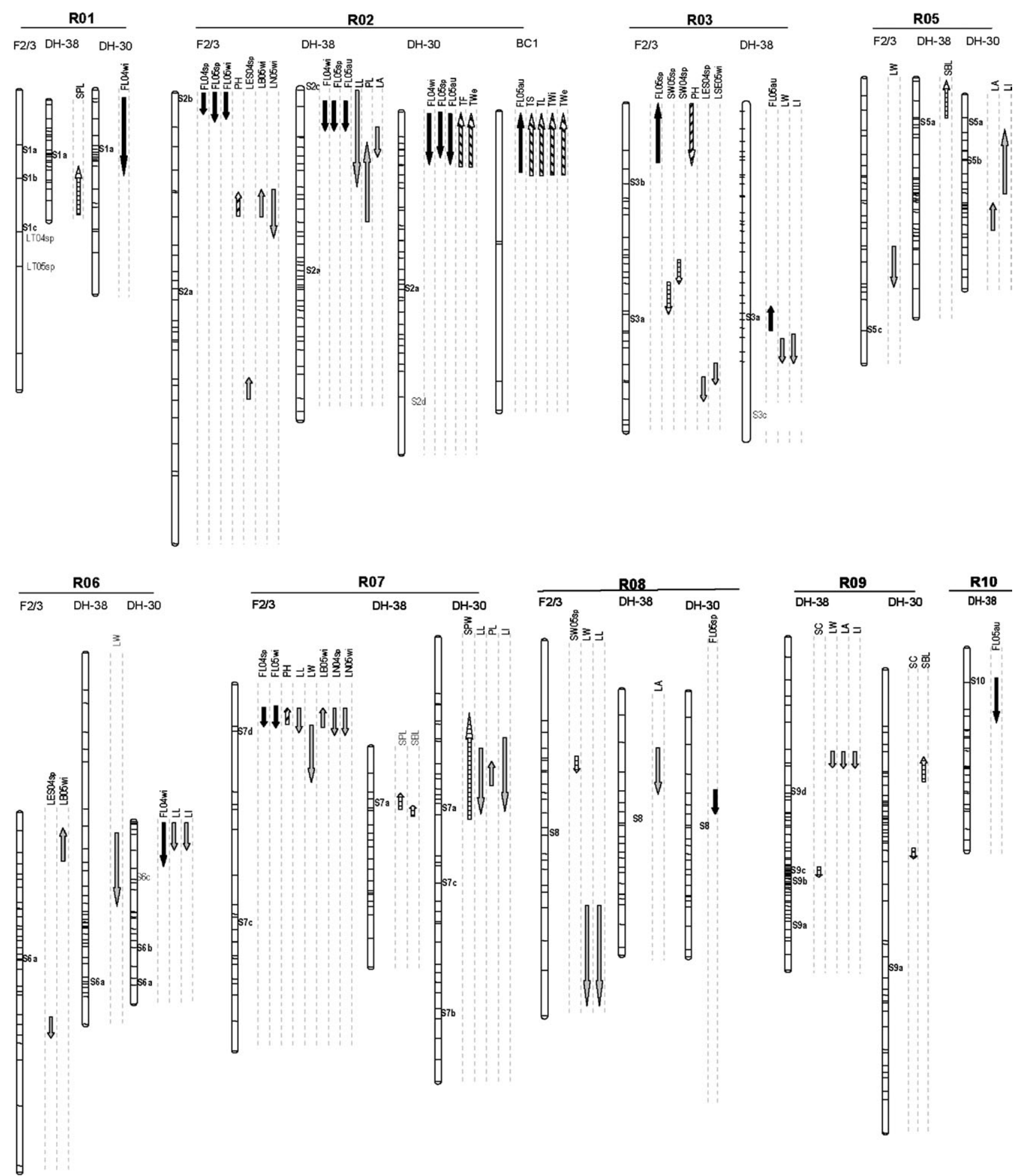

Fig. 3. Locations of QTL for the traits analysed in the four mapping populations. The linkage groups of different maps are aligned based on common SSR (S1-S10) or common AFLP markers (P Lou et al., unpublished data). The lengths of the arrows indicate the 2-LOD support intervals. The traits (see abbreviations in Table 2) are indicated above each column. The direction of the arrow's head indicates the allelic effect: upward, RC-144 increases and CC-156 decreases for $\mathrm{F}_{2 / 3}$, YS-143 increases and PC-175/VT-115 decreases in DH-38 and DH-30; downward: CC-156 increases and RC-144 decreases, YS-143 decreases and PC-175/VT-115 increases in DH-38 and DH-30. The filling pattern of arrows refers to different groups of phenotypic traits. Flowering time; seed; plant height; leaf; turnip. S1a, BRMS096; S1b, Ra2G09; S1c, BRMS037; S2a, Na12H09; S2b, BrFLC2; S2c KS50030; S2d, BrMAF; S3a, BRMS043; S3b, BRMS042; S3c, BrFLC3; S5a, BRMS034; S5b, BRMS007; S5c, Ra3H10; S6a, BRMS014; S6b, Na12H07; S6c, KS51082; S7a, BRMS018; S7b, O112E03; S7c, Ra2A01; S7d, BRMS036; S8, Ra2E12; S9a, BRMS051; S9b, Na10A08; S9c, O112F02; S9d, O110D08; S10, BrFLC1. 
these loci $\mathrm{CC}-156$ and $\mathrm{PC}-175$ contributed the earlier flowering time alleles.

Seed and seedpod traits: Four QTL were detected for seedpod traits (SPQTLn) and one QTL for seed coat colour (SCQTL-1) in the two DH populations, and two QTL for seed weight (SWQTLn) in the RC-CC $\mathrm{F}_{2 / 3}$ population. The proportion of total variation explained by each QTL ranged from $11.1 \%$ to $38.1 \%$ for seed pod traits, $61.7 \%$ to $65.5 \%$ for seed coat colour, and $10.2 \%$ to $17.6 \%$ for seed weight (Table 5; Fig. 3).

Two genomic regions, on R05 and R09, each harboured a single seedpod trait (SBL) in DH-38, and one genomic region on R07 affected three seedpod traits (SPL and SBL in DH-38, SPW in DH-30). In both the DH-38 and DH-30 population, one major genomic region on R09 affected seed coat colour (SC) with a high explained phenotypic variation $(>60 \%)$ and LOD value (>10.0), indicating an almost monogenic inheritance of the yellow seed trait derived from YS-143. In RC-CC $F_{2 / 3}$ one genomic region in the middle of R03 affected seed weight (SW) and was detected in the 2004 and 2005, with the RC-144 allele decreasing seed weight.

Growth-related and leaf traits: Three QTL affecting plant height (PHQTLn) were detected on R02, R03, and R07 in the $\mathrm{RC}-\mathrm{CC} \mathrm{F}_{2 / 3}$ population, explaining 23.9, 15.7, and $8.9 \%$ of the phenotypic variation respectively. Two markers, E33M51-7CC and BRMS037, $10 \mathrm{cM}$ apart on R01, were linked with the number of leaf trichomes (LT04sp and LT05sp). QTL for the number of branches (PB) in DH-30 and BC1 populations could not be detected.

Ten QTL for leaf traits (LQTLn), distributed over seven linkage groups, were detected in the $\mathrm{F}_{2 / 3}$ and $\mathrm{DH}$ populations; the proportion of total variation explained by each QTL ranged from 6.5\% to $26.4 \%$ (Table 5). The five different parents contributed alleles with effects in both directions to most of these traits (Fig. 3). Seven genomic regions affected two or more leaf traits, where LW cosegregated with other leaf traits (LL, LA, LI, LN or LB). LQTL-1 on R02, LQTL-3 on R03, LQTL-4 on R05, LQTL-5 on R06, and LQTL-7 on R07 were detected in multiple populations, related to multiple traits, and appeared to be the major QTL affecting leaf size in the used populations. Two genomic regions (LQTL-2 on R02 and LQTL-6 on R06) affected only leaf edge shape (LES04sp) and represent loci for leaf serration. It is hard to conclude whether LQTL-8 on R08 maps in the same region as LQTL-9 on R08 because only one common SSR marker connects the R08 maps of $\mathrm{F}_{2 / 3}$ and DH-38.

\section{Turnip formation}

One major QTL for turnip-related traits (TuQTL-1) was detected both in the $\mathrm{DH}-30$ and the $\mathrm{BC} 1$ population (Table 5; Fig. 3) and this QTL co-located with FLQTL-2 on the top of R02. In the BC1 population the TuQTL-1 explained about $24.0 \%$ of variation and in DH-30 this QTL explained $36.7-40.0 \%$ of the variation.

\section{Clustering of QTL}

Several QTL positions were detected, where one locus controlled multiple traits, and which are possibly physiologically related. Many QTL co-localized at the top of R02, mainly for flowering time, but also for leaf traits in the $\mathrm{F}_{2 / 3}$ and for turnip formation in both the DH-30 and BC1 population (Fig. 3). Clusters of QTL were also detected in the middle of R06 and above the middle of R07. Clustering of QTL was consistent with the strong genetic correlations observed among specific traits (e.g. turnip and flowering traits, Fig. 2), which is also obvious from the PCA for the traits that have significant loadings for the respective PCA component.

\section{Discussion}

\section{Flowering time}

In this study, QTL were mapped for flowering time in four different populations derived from crosses between diverse parental morpho-types. This multiple population approach has the advantage that alleles of five parental accessions can be evaluated and revealed a large number of genomic regions harbouring allelic variation for flowering time. Eight possible genomic regions on seven linkage groups affected flowering time, two of them (FLQTL-2, FLQTL-6) were detected in different conditions, suggesting that they are not, or only marginally, affected by the environment. Some of the flowering-related QTL (FLQTLn) that were found co-localized with previously published QTL detected in other B. rapa populations. In Fig. 4, maps of linkage groups are depicted with the map positions of flowering-time-related genes and with positions of QTL identified in this study.

\section{Role of FLC paralogues in regulation of flowering time}

A number of $F L C$ paralogues (BrFLC1, BrFLC2, $B r F L C 3$, and BrFLC5) were mapped in this and previous studies (Schranz et al., 2002; Kim et al., 2006). In previous QTL analyses of flowering time in Brassica, evidence has been presented for a role of FLCs as candidate genes underlying flowering time in B. napus, $B$. oleracea, and B. rapa (Osborn et al., 1997; Schranz et al., 2002; Okazaki et al., 2007). CO and FLC are linked on Arabidopsis chromosome 5 (15 cM apart) and based on the synteny between Arabidopsis and Brassica (Parkin et al., 2005; Schranz et al., 2006) they are linked in Brassica as well. In other studies, $C O$ and COL1 are mentioned as candidate genes underlying flowering time QTL in B. nigra (Lagercrantz et al., 2002; Osterberg 


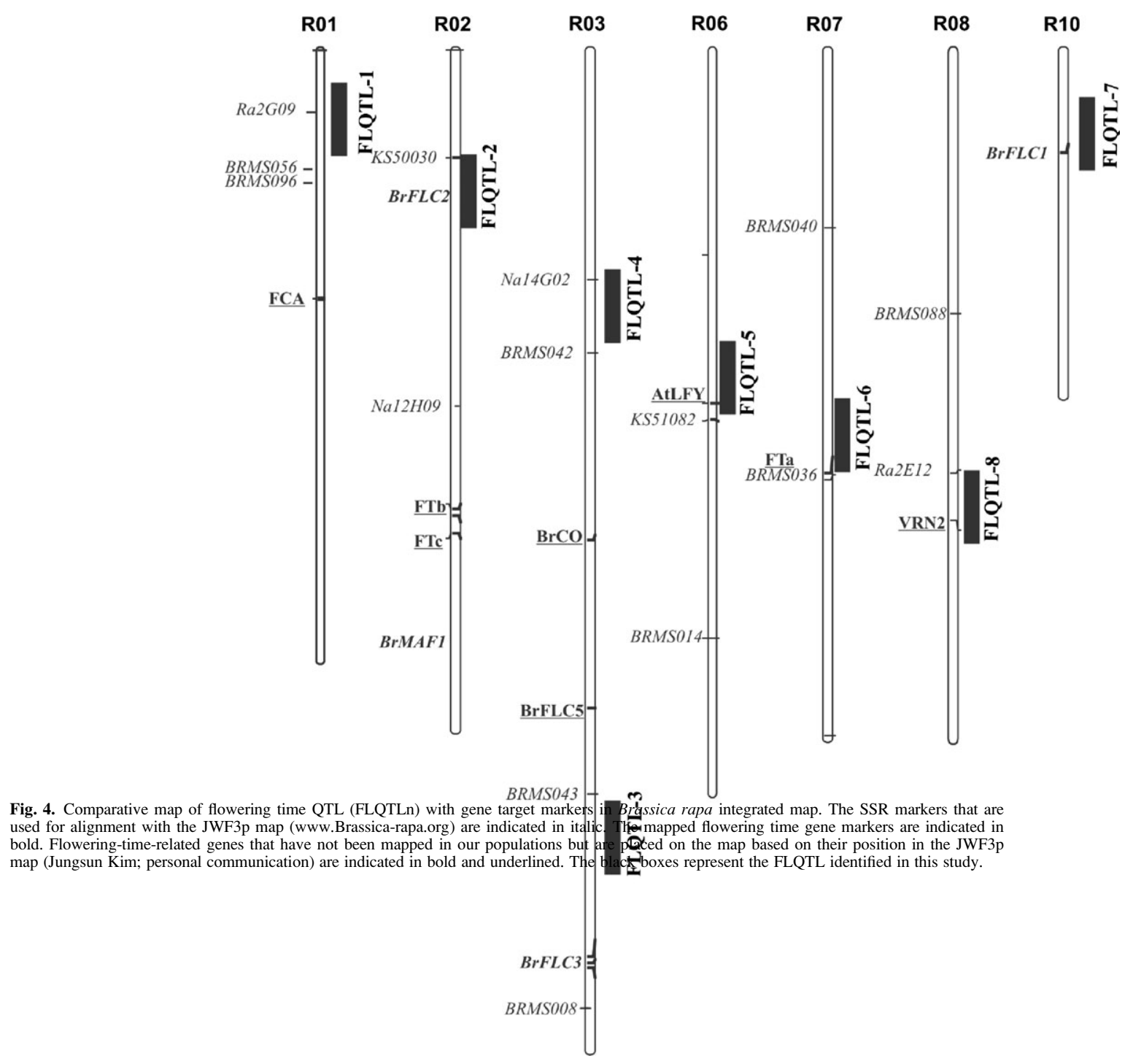

et al., 2002). Axeisson et al. (2001) identified flowering time QTL in B. oleracea, B. juncea, B. nigra, and B. rapa and mapped the candidate genes $C O$ and $F L C$. Their data were consistent with a role for duplicated copies of the ancestral genes as candidates underlying flowering time QTL and they suggested the $C O$ gene as the candidate (Axeisson et al., 2001).

The use of SSR markers allowed the alignment of our maps to the B. rapa reference maps and to compare QTL positions between populations. Our data suggest that several of the flowering time loci correspond to the map positions of $F L C$ paralogues. Four FLC homologues, BrFLC1, BrFLC2, BrFLC3, and BrFLC5, have been cloned in B. rapa, and mapped using in situ hybridization, and genetic mapping with AFLPs or linked SSR markers (Kim et al., 2006; Yang et al., 2006). BrFLC2 is mapped on the top of R02 and BrFLC3 ( $a$ and $b$ ) is mapped on the bottom of R03 between SSR marker BRMS043 and BRMS008. BrFLC2 was located on R02 using SSR markers in the $\mathrm{F}_{2 / 3}$, DH-38, and DH-30 populations, and co-localized with the major FLQTL-2 on R02. BRFLC5 was mapped on the lower middle of R03, $33 \mathrm{cM}$ from $B r F L C 3$. BrFLC1 was mapped on the top of $\mathrm{R} 10$ at the same position as FlQTL-7 identified in DH38 only in autumn 2005.

FLQTL-4 locates at the top of R03 in the RC-CC $\mathrm{F}_{2 / 3}$ population, which is the position of the FR2 flowering time QTL (Osborn et al., 1997), co-localizing with 
BrFLC5 (Schranz et al., 2002). The orientation of the different maps compared to the JW3p reference map was based on a number of SSRs. However, only one SSR (BRMS043) was common between the $\mathrm{F}_{2 / 3}$ and the DH populations. The position of FLQTL-4 relative to FLQTL-3 both on R03 could not be determined and therefore BrFLC5 may not be a candidate for FLQTL-4. FLQTL-3 maps between $B r F L C-5$ and $B r-F L C-3$ on $\mathrm{R} 03$, at a synthetic position compared to a $B$. oleracea flowering time QTL identified by Okazaki et al. (2007).

FLQTL-2 colocalizing with FLC2 on R02 determines the flowering differences between the early oil types and the other middle late morpho-types. Loci that are detected only in some of the populations that were studied here represent loci that differ between the different parental vegetable types used. This QTL also shows a significant genotype $\times$ environment interaction because the explained variance was much lower after vernalization (data not shown) and when plants did grow under low temperature field conditions. This is in agreement with the described effects on FLC of which the expression is reduced by cold (Koornneef et al., 2004). Schranz et al. (2002) also found for the FR-1 locus, which most likely is the same as FLQTL-2, that it explains more variation without vernalization.

\section{Role of other flowering-time-related genes}

In Arabidopsis, a number of additional flowering-related genes were described and were shown to interact in a network. Examples are FT (FLOWERING LOCUS T), $L F Y$ (LEAFY), and VNR2 (VERNALIZATION2). Colocalization of flowering time QTL with flowering-related genes renders them candidate genes for the FLQTL. In $B$. rapa, one FT paralogue was recently mapped on R07 (BrFTa) close to SSR marker BRMS036 (Jungsun Kim; personal communication); FLQTL-6 in $\mathrm{F}_{2 / 3}$ maps near this SSR marker BRMS036 on R07 (S7d, Fig. 4). One $L F Y$ paralogue has been mapped on the lower middle of R06 (Kim et al., 2006) while also FLQTL-5 in DH-30 was mapped on the middle of R06. Lack of common SSRs in this region makes map comparison of FLQTL-5 and $B r L F Y$ not possible. FLQTL-8 co-localizes with VRN2, a gene in the vernalization response pathway.

\section{Turnip formation}

In the present study, a single QTL for each of the traits turnip width, weight, and length was detected at the top of R02 which co-localizes with the major flowering time QTL (FLQTL-2). This co-localization of QTL can either be explained by tight linkage or pleiotropy, or by epistasy of flowering time over turnip formation because a plant that flowers early allocates its energy to flower formation and developing seeds, while turnip formation requires the redirection of most assimilates to the roots.
Other morphological traits and genetic architecture of trait variation

Besides flowering time and turnip formation, a genetic analysis of other morphological traits is provided. A number of QTL underlying these traits in the populations are observed at many loci throughout the whole genome. Typically, the parental accessions contained alleles that both increased and decreased leaf phenotypes, resulting in large transgressive segregation within the population.

Co-location of QTL for phenotypic traits are found in many cases, indicating that these loci may have an overall effect on plant development and suggest a pattern of genetic integration of morphological traits. For example, the genomic regions at the top of R02 and the higher middle of R07 affect seed traits, growth-related traits, leaf traits, flowering time, and turnip formation, in which multiple linked genes or pleiotropic loci controlling related developmental characteristics may be involved. On the other hand LQTL-5 on R06 was identified across all the populations affecting specifically leaf traits (LB05wi, LW, LL, and LI). QTL for leaf serration (LES) did not colocalize with other leaf traits (LL, LW, LA, and LI), suggesting independent inheritance. This was also the case for the seed colour QTL (SCQTL), mapping near SSR markers, Na10A08 and O112F02, of which Na10A08 showed a strong association with seed coat colour in Brassica juncea (Padmaja et al., 2005).

The coincidence of QTL locations generally supports the observed phenotypic correlations, such as flowering time and turnip formation mentioned above. The QTL clusters in this study, such as the genomic regions on R02, R03, R06, and R07, reflect the genetic correlations between the traits studied. Presently more floral traits (petal and sepal development) are being measured to investigate the correlation between floral and leaf traits further, for which in Arabidopsis a weak correlation was observed and differentiation between floral and vegetative modules was suggested (Juenger et al., 2000).

\section{Supplementary data}

Supplementary data are available at $J X B$ online in Table S1 giving a list of all the PCA and QTL analysis results of flowering time and morphological traits in all the populations.

\section{Acknowledgements}

We thank Nurmi PD Pangesti, Yao Wang, and Jiangling Xiong for phenotypic scoring of turnip characteristics, testing of SSR markers, and constructing the $\mathrm{BC} 1$ genetic map. We thank Liu Nini for phenotypic scoring of the DH populations after vernalization. We are grateful to staff in the Plant Science Experimental Centre of Wageningen University, the Netherlands, and Yanguo Zhang and Yanling Liu in the Institute of Vegetables and Flowers (Beijing), Chinese Academy of Agriculture Science, China, for taking care of 
the plants. The project is sponsored by the Royal Dutch Academy of Sciences (KNAW), the Asian Facility (project AF01/CH/8 'SinoDutch Genomic Lab and Vegetable Research Center') and a fellowship from an exchange student programme (Huygens) between the Chinese Scholarship Council and Nuffic in the Netherlands.

\section{References}

Ajisaka H, Kuginuki Y, Yui S, Enomoto S, Hirai M. 2001. Identification and mapping of a quantitative trait locus controlling extreme late bolting in Chinese cabbage (Brassica rapa L. ssp. pekinensis syn. campestris L.) using bulked segregant analysis. Euphytica 118, 75-81.

Axeisson T, Shavorskaya O, Lagercrantz U. 2001. Multiple flowering time QTLs within several Brassica species could be the result of duplicated copies of one ancestral gene. Genome 44, 856-864.

Bohuon EJ, Ramsay LD, Craft JA, Arthur AE, Marshall DF, Lydiate DJ, Kearsey MJ. 1998. The association of flowering time quantitative trait loci with duplicated regions and candidate loci in Brassica oleracea. Genetics 150, 393-401.

Conner JK. 2002. Genetic mechanisms of floral trait correlations in a natural population. Nature 420, 407-410.

Engelmann K, Purugganan M. 2006. The molecular evolutionary ecology of plant development: flowering time in Arabidopsis thaliana. Advances in Botanical Research 44, 507-526.

Juenger T, Purugganan M, Mackay TF. 2000. Quantitative trait loci for floral morphology in Arabidopsis thaliana. Genetics 156, 1379-1392.

Kennard W, Slocum M, Figdore S, Osborn T. 1994. Genetic analysis of morphological variation in Brassica oleracea using molecular markers. Theoretical and Applied Genetic 87, 721-732.

Kim JS, Chung TY, King GJ, Jin M, Yang TJ, Jin YM, Kim HI, Park BS. 2006. A sequence-tagged linkage map of Brassica rapa. Genetics 174, 29-39.

Kole C, Quijada P, Michaels SD, Amasino RM, Osborn TC. 2001. Evidence for homology of flowering-time genes VFR2 from Brassica rapa and FLC from Arabidopsis thaliana. Theoretical and Applied Genetics 102, 425-430.

Koornneef M, Alonso-Blanco C, Vreugdenhil D. 2004. Naturally occurring genetic variation in Arabidopsis thaliana. Annual Review of Plant Biology 55, 141-172.

Lagercrantz U, Kruskopf Osterberg M, Lascoux M. 2002. Sequence variation and haplotype structure at the putative flowering-time locus COL1 of Brassica nigra. Molecular Biology Evolution 19, 1474-1482.

Lan TH, Paterson AH. 2000. Comparative mapping of quantitative trait loci sculpting the curd of Brassica oleracea. Genetics 155, 1927-1954.

Nishioka M, Tamura K, Hayashi M, Fujimori Y, Ohkawa Y, Kuginuki Y, Harada K. 2005. Mapping of QTL for bolting time in Brassica rapa (syn. campestris) under different environmental conditions. Breed Science 55, 127-133.

Okazaki K, Sakamoto K, Kikuchi R, Saito A, Togashi E, Kuginuki Y, Matsumoto S, Hirai M. 2007. Mapping and characterization of $F L C$ homologs and QTL analysis of flowering time in Brassica oleracea. Theoretical and Applied Genetics 114, 595-608.

Osborn TC, Kole C, Parkin IA, Sharpe AG, Kuiper M, Lydiate DJ, Trick M. 1997. Comparison of flowering time genes in Brassica rapa, B. napus, and Arabidopsis thaliana. Genetics 146, 1123-1129.
Osterberg MK, Shavorskaya O, Lascoux M, Lagercrantz U. 2002. Naturally occurring indel variation in the Brassica nigra COLl gene is associated with variation in flowering time. Genetics 161, 299-306.

Padmaja KL, Arumugam N, Gupta V, Mukhopadhyay A, Sodhi YS, Pental D, Pradhan AK. 2005. Mapping and tagging of seed coat colour and the identification of microsatellite markers for marker-assisted manipulation of the trait in Brassica juncea. Theoretical and Applied Genetics 111, 8-14.

Parkin IA, Gulden SM, Sharpe AG, Lukens L, Trick M, Osborn TC, Lydiate DJ. 2005. Segmental structure of the Brassica napus genome based on comparative analysis with Arabidopsis thaliana. Genetics 171, 765-781.

Rae AM, Howell EC, Kearsey MJ. 1999. More QTL for flowering time revealed by substitution lines in Brassica oleracea. Heredity 83, 586-596.

Schranz ME, Lysak MA, Mitchell-Olds T. 2006. The ABC's of comparative genomics in the Brassicaceae: building blocks of crucifer genomes. Trends in Plant Science 11, 535-542.

Schranz ME, Quijada P, Lukens L, Osborn TC, Sung SB, Amasino R. 2002. Characterization and effects of the replicated flowering time gene FLC in Brassica rapa. Genetics 162, 1457-1468.

Sebastian RL, Kearsey MJ, King GJ. 2002. Identification of quantitative trait loci controlling developmental characteristics of Brassica oleracea L. Theoretical and Applied Genetics 104, 601-609.

Song K, Slocum MK, Osborn TC. 1995. Molecular marker analysis of genes controlling morphological variation in Brassica rapa (syn. campestris). Theoretical and Applied Genetics 90, $1-10$.

Suwabe K, Tsukazaki H, Iketani H, Hatakeyama K, Kondo M, Fujimura M, Nunome T, Fukuoka H, Hirai M, Matsumoto S. 2006. Simple sequence repeat-based comparative genomics between Brassica rapa and Arabidopsis thaliana: the genetic origin of clubroot resistance. Genetics 173, 309-319.

Teutonico RA, Osborn TC. 1994. Mapping of RFLP and quantitative trait loci in Brassica rapa and comparison to the linkage maps of B. napus, B. oleracea, and Arabidopsis thaliana. Theoretical and Applied Genetics 89, 885-894.

Van Ooijen JW. 2004. MAPQTL5, Software for mapping of quantitative trait loci in experimental populations. Plant Research International Wageningen, the Netherlands.

Van Ooijen JW, Voorrips RE. 2001. Joinmap version 3.0: software for the calculation of genetic linkage maps. Plant Research International, Wageningen, the Netherlands.

Voorrips RE. 2002. MapChart: Software for the graphical presentation of linkage maps and QTLs. The Journal of Heredity 93, $77-78$.

Yang TJ, Kim JS, Kwon SJ, et al. 2006. Sequence-level analysis of the diploidization process in the tiplicated FLOWERING LOCUS C region of Brassica rapa. The Plant Cell 18, 13391347.

Yu SC, Wang YJ, Zheng XY. 2003. Mapping and analysis QTL controlling some morphological traits in Chinese cabbage (Brassica campestris L. ssp. pekinensis). Acta Genetic Sinia 30, 1153-1160.

Zhang X, Wu J, Zhao J, et al. 2006. Identification of QTLs related to bolting in Brassica rapa ssp. pekinensis. Agricultural Sciences in China 5, 265-271.

Zhao J, Wang X, Deng B, Lou P, Wu J, Sun R, Xu Z, Vromans J, Koornneef M, Bonnema G. 2005. Genetic relationships within Brassica rapa as inferred from AFLP fingerprints. Theoretical and Applied Genetics 110, 1301-1314. 